\title{
Isomorph invariance of dynamics of sheared glassy systems
}

\author{
Yonglun Jiang $\odot$ and Eric R. Weeks $\odot$ \\ Department of Physics, Emory University, 400 Dowman Drive, Atlanta, Georgia 30322, USA \\ Nicholas P. Bailey (e) \\ Glass and Time, IMFUFA, Department of Science and Environment, Roskilde University, P.O. Box 260, 4000 Roskilde, Denmark
}

(Received 31 July 2019; published 25 November 2019)

\begin{abstract}
We study hidden scale invariance in the glassy phase of the Kob-Andersen binary Lennard-Jones system. After cooling below the glass transition, we generate a so-called isomorph from the fluctuations of potential energy and virial in the $N V T$ ensemble: a set of density, temperature pairs for which structure and dynamics are identical when expressed in appropriate reduced units. To access dynamical features, we shear the system using the SLLOD algorithm coupled with Lees-Edwards boundary conditions and study the statistics of stress fluctuations and the particle displacements transverse to the shearing direction. We find good collapse of the statistical data, showing that isomorph theory works well in this regime. The analysis of stress fluctuations, in particular the distribution of stress changes over a given strain interval, allows us to identify a clear signature of avalanche behavior in the form of an exponential tail on the negative side. This feature is also isomorph invariant. The implications of isomorphs for theories of plasticity are discussed briefly.
\end{abstract}

DOI: 10.1103/PhysRevE.100.053005

\section{INTRODUCTION}

Recently, it was discovered that a broad class of classical condensed matter systems exhibit an approximate scale invariance [1-6]. Upon changing a system's density, a corresponding change in temperature can be found such that the structure and dynamics of the system are unchanged, as long as they are compared in an appropriate dimensionless form. State points which are equivalent in this sense are said to be isomorphic, and the key feature of systems exhibiting so-called hidden scale invariance is the existence of isomorphic curves, or isomorphs, in the phase diagram [5]. The theory of isomorphs shows how they can be identified straightforwardly in computer simulations, how to appropriately scale quantities for comparison, and which quantities are expected to be isomorph invariant. Isomorphs have been identified and investigated in the equilibrium liquid state for many model systems [1,710]. Systems with good isomorphs include those dominated by van der Waals interactions, including molecular systems, and most metals [11], while strong directional bonds, as in hydrogen-bonding systems and network formers, generally give rise to more complex behavior and the absence of isomorphs. Water is a good example of a system without good isomorphs. The phase is not important for whether isomorphs can be found, as long as relatively-high-density condensed phases are considered. Nor is equilibrium essential; isomorphs have been studied in conditions of nonequilibrium steadystate shearing [12] and aging [5,13,14] and zero-temperature shearing of a glass [15]. The class of systems exhibiting good isomorphs has been referred to as R-simple systems (R denoting Roskilde). For reviews, the reader may consult Refs. $[7,16,17]$. In the present work isomorphs are investi- gated in the context of deformation of the glass state at finite temperature.

We consider an amorphous solid created by cooling a viscous liquid down below its glass transition and then applying Couette-type shearing at constant volume and fixed strain rate. This necessarily involves a departure from equilibrium and in principle introduces a potential dependence on history, for example, through the cooling rate, as well as possible aging effects, into the system's behavior. We minimize these issues by restricting our attention to steady-state shearing: If one shears the system at a constant strain rate beyond, say, 0.5 or 1.0 strain, a steady state is obtained which depends only on the density, the temperature, and the strain rate. As discussed in Ref. [12], the existence of isomorphs reduces these three variables to two: a variable labeling the isomorph (in equilibrium this is generally taken to be the excess entropy) and a dimensionless strain rate. In principle, however, isomorph theory allows for independent configurations from equilibrium states above the glass transition which are isomorphic to each to be cooled into the glassy state in an isomorphic way such that the entire thermal histories and deformation histories are isomorphic. In that case the entire stress-strain curves could be compared, rather than simply the steady-state part. Some ten years ago Lerner and Procaccia proposed a scaling theory for steady-state plasticity based on approximating the pair potential by an inverse power law [18]. The relation between that work and isomorph theory will be discussed below.

We work with the usual Kob-Andersen binary LennardJones glass forming model [19-21], which is useful because it is difficult (though not impossible [22,23]) to crystallize on computer timescales. It is certainly straightforward to obtain a glassy state in a simulation with sufficiently rapid cooling. 
We consider two starting states in the glass, one just below the glass transition and one deep in the glass. The focus of the analysis is on analyzing steady-state stress-strain curves statistically and the particle displacements characterized by the mean-square displacement.

Demonstrating the presence of good isomorphs in the glassy state has theoretical relevance not just because it permits a simplification of the phase diagram, but for two other reasons. First, given the existence of isomorphs, it becomes clearer what the relevant thermodynamic variables are: Pressure, while being of course extremely relevant from an experimental point of view, becomes secondary to density. Moreover, strain rates should be specified and compared in their dimensionless (reduced) form. Second, the existence of isomorphs puts a strong constraint on theories of glassy behavior. Several theories for the mechanical properties of amorphous materials have been proposed. Hidden scale invariance imposes constraints on candidate theories, since a theory which purports to be general should in particular apply to systems with hidden scale invariance and should therefore involve equations expressed in reduced-unit quantities which are explicitly isomorph invariant. This principle has been called the isomorph filter [5,24]. In the context of theories of the glass transition, for example, a theory connecting the (reduced) relaxation time to the configurational entropy, with no other dependence on thermodynamic state, passes the isomorph filter because both quantities are isomorph invariant. It should be noted that thermodynamic quantities such as the average potential energy, pressure, and bulk modulus, and thereby the equation of state, are not isomorph invariant; interaction terms can be added to a model which hardly fluctuate in a bulk system at fixed density, which can thus affect the equation of state but not the structure and dynamics.

The following section gives an overview of the most essential results from the theory of isomorphs. Section III then describes the system and the simulation methods used. Section IV describes how we generated glassy isomorphs and checks the isomorph invariance of their structure using the radial distribution function. The main analysis of the paper is presented in the following two sections: Sec. V presents a detailed analysis of the stress-strain curves while Sec. VI contains an analysis of particle displacements via the mean-square displacement transverse to the shearing direction. Section VII discusses implications of the existence of isomorphs for theories of plasticity, showing via an example from the literature how density dependence can be included in an isomorph invariant way. Section VIII summarizes and concludes the paper.

\section{ISOMORPH THEORY}

In this section we give a brief overview of the theoretical basis for analyzing isomorphs, starting with how to put observables in the necessary dimensionless forms needed to properly compare structure and dynamics at different thermodynamic state points.

\section{A. Reduced units}

As mentioned above, quantities must be expressed in an appropriate dimensionless form, referred to as using reduced units. We essentially scale out the direct effects of changing density and temperature on structure and dynamics: If we have $N$ particles in a volume $V$, then the system's (number) density is $\rho \equiv N / V$. A basic length scale $l_{0}$ is defined by interparticle spacing $\rho^{-1 / 3}$. If the system is in equilibrium at temperature $T$, then a basic timescale is defined by the time for a particle with the thermal velocity $\sqrt{k_{B} T / m}$ to cover a distance $l_{0}$ : $t_{0}=\rho^{-1 / 3}\left(k_{B} T / m\right)^{-1 / 2}$. In the case of a mixture, the average mass $\langle m\rangle$ should be used. Given $l_{0}$ and $t_{0}$, we can rescale space and time, making it possible, for example, to compare trajectories at different state points; the rescaling accounts for the most trivial effects of changing density and temperature. In fact, all physical quantities can be rescaled similarly, by taking appropriate combinations of $l_{0}, t_{0}$, and $\langle m\rangle$. For a quantity with dimensions of energy, the scale factor is just $k_{B} T$. For a pressure (or stress or elastic modulus) the scale factor is $\rho k_{B} T$. We denote the rescaled reduced-unit quantities by a tilde; thus the reduced form of a particle position $\mathbf{r}$ is $\tilde{\mathbf{r}} \equiv \rho^{1 / 3} \mathbf{r}$.

\section{B. Identifying isomorphs}

The scale invariance that underlies the existence of isomorphs derives ultimately from the fact that the potential energy surface of the $N$-particle system changes in a somehow homogeneous way when density is changed. For example, suppose changing the density of any microscopic configuration by a factor $\lambda$ results in the potential energies being changed by a factor $\lambda^{\gamma}$ for some exponent $\gamma$. This can then be compensated by increasing temperature by the same factor, meaning all Boltzmann factors will be unchanged, so the statistical probability of all microstates will be the same at the new density as for the corresponding unscaled configurations at the original density. It follows that all statistical measures of structure will be invariant when expressed in terms of the reduced coordinates $\tilde{\mathbf{r}}$. It can also be shown [4] that the equation of motion is also the same for both states when expressed in reduced units and therefore that all dynamical quantities are also invariant in reduced units. The case just described is realized by systems interacting with an inverse power law pair potential $v=A / r^{n}$; in that case the scaling exponent $\gamma$ is given by $n / 3$. In that case isomorphs are exact, trivial, and well known and the invariant quantities include not just all structural and dynamical quantities but also thermodynamic quantities in reduced units. More generally, we do not expect to find exact isomorphs, but we find very good approximations. The simplest way to express and identify hidden scale invariance was shown in Ref. [25], where the essential condition was stated as follows: A change of density must preserve the order of potential energies of microstates. To test for scale invariance, we consider infinitesimal changes of density under uniform scaling, whereupon changes in the potential energies $U$ of microstates are given by

$$
d U=W d \ln \rho
$$

here $W$ is the virial, a quantity typically calculated in computer simulations due to its appearance in the formula for pressure [26]. Requiring that the order of energies be preserved means in particular that configurations at a given density with the same $U$ will experience the same change in $U$ upon an infinitesimal change of $\rho$. By Eq. (1) this means they have 
the same $W$. In other words, the potential energy and virial must be strongly correlated (the discovery of strong $U$ and $W$ correlations [27] marked the beginning of the development of isomorph theory). Linear regression applied to a scatter plot of $W$ versus $U$ yields two parameters, namely, the correlation coefficient

$$
R=\frac{\langle\Delta U \Delta W\rangle}{\sqrt{\left\langle(\Delta U)^{2}\right\rangle} \sqrt{\left\langle(\Delta W)^{2}\right\rangle}}
$$

and the slope

$$
\gamma=\frac{\langle\Delta U \Delta W\rangle}{\left\langle(\Delta U)^{2}\right\rangle} .
$$

Here angular brackets denote canonical ensemble averages and $\Delta X \equiv X-\langle X\rangle$ for any quantity $X$. A value of $R$ close to unity in a region of the phase diagram (typically values above around 0.9 are considered good, although lower thresholds have also been used [11]) indicates that the system exhibits hidden scale invariance and should have good isomorphs in that part of the phase diagram. The interpretation of the slope $\gamma$ was given in Ref. [5]: It is the slope of curves of constant excess entropy (that is, configurational adiabats) in the $(\ln \rho, \ln T)$ phase diagram

$$
\left(\frac{\partial \ln T}{\partial \ln \rho}\right)_{S_{\mathrm{ex}}}=\gamma(\rho, T) .
$$

The excess entropy is defined as the entropy minus that of the ideal gas with the same density and temperature and is one of the thermodynamic properties which is invariant along an isomorph. Thus, in systems with good isomorphs, the $\gamma$ of Eq. (3) is just the density scaling exponent $\gamma$ discussed above. In the Schrøder-Dyre formulation of the theory, the status of configurational adiabats was raised such that these are considered to define isomorphs in systems with strong $U$ and $W$ correlations [25]. Since $\gamma(\rho, T)$ can be calculated at any state point using the fluctuation formula (3), Eq. (4) provides a general method to generate isomorphs by numerical integration. Typically, steps of order $1 \%$ in density are used.

\section{SIMULATIONS}

The system studied is the usual binary Lennard-Jones system introduced by Kob and Andersen [19-21], which has been mainly studied at one particular density $1.2 \sigma_{A A}^{-3}$ (where the $A$ particles are the larger ones). From now on, when not using reduced units, we work with the unit system defined by the Lennard-Jones (LJ) parameters of the $A$ particles' interactions with each other, $\sigma_{A A}$ and $\epsilon_{A A}$, and the mass which is the same for both $A$ and $B$ particles; thus, temperature is given in units of $\epsilon_{A A} / k_{B}$. The potential is cut off using the shifted-force method [28] at $2.5 \sigma$ for each type of interaction. The number of particles is 1000 (with the usual composition of $80 \% \mathrm{~A}$ ). The simulations are carried out on a GPU cluster using the RUMD software [29,30].

The glassy states are created by cooling a liquid at constant pressure at a fixed cooling rate from temperature $T=1.0$ down to a given start temperature. Different cooling rates are applied, but for the steady-state results presented in this work the cooling rate is not relevant. The reason for cooling at fixed pressure rather than fixed volume is to avoid arriving at a state where the pressure is very low or negative, since good

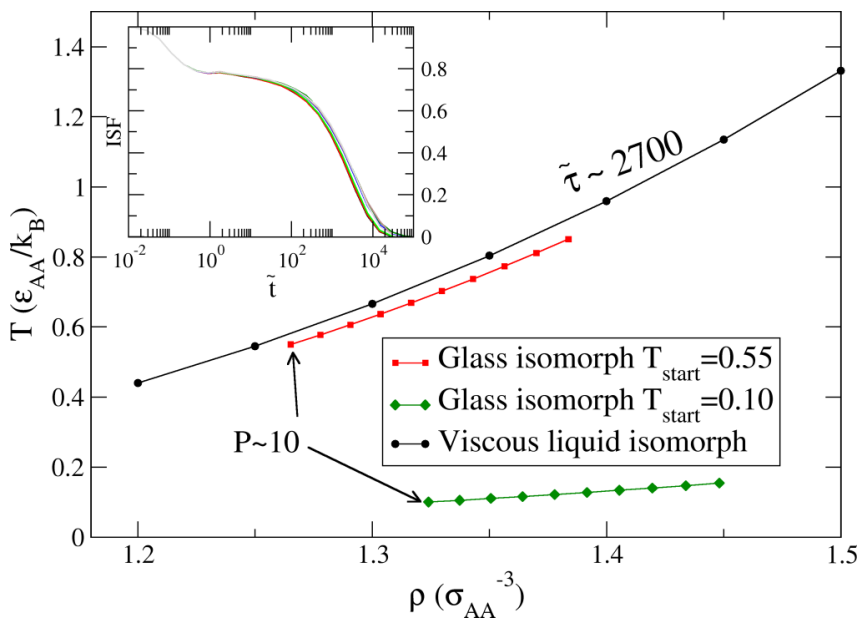

FIG. 1. Black symbols indicate an isomorph in the supercooled liquid which includes the point $(\rho=1.2, T=0.44)$. We use this as a guide to locating the glass transition; its relaxation time is about 2700 in reduced units, corresponding to 3850 in $\mathrm{LJ}$ units at the lowest density 1.2. The inset shows the intermediate scattering function for the different state points, lying almost on top of each other. The red and green symbols indicate isomorphs generated in the glass which we use for studying deformation, referred to as those starting at temperatures $T=0.55$ and 0.1 , respectively. Note that the starting densities are not the same, since these are taken from a cooling run at fixed pressure $P=10$.

isomorphs are generally obtained at not too low pressures. To locate our glassy isomorphs in the phase diagram and compare to other work on this system, it is useful to have an idea of where the glass transition is. When considering the full phase diagram the glass transition can be defined as the set of $(\rho, T)$ points where the liquid's relaxation time attains some fixed value. There are two sources of ambiguity or arbitrariness in such a definition: which observable to use when defining the relaxation time and which value to set as defining $T_{g}(\rho)$. Experimentally, for the latter one chooses conventionally a value of order $100 \mathrm{~s}$ in real units; with the isomorph theory in mind it is natural to specify a criterion in reduced units, since in a system with isomorphs the glass line will then correspond to an isomorph [13]. In computer simulations, relaxation times of order $100 \mathrm{~s}$ are nowhere near realistic, so as a guide we choose a viscous liquid state which can be equilibrated in reasonable time. In Fig. 1 we plot a viscous liquid isomorph whose temperature at the usual Kob-Andersen density 1.2 is 0.44 . The relaxation time there (based on fitting the self-intermediate scattering function of the $A$ particles to a stretched exponential function) is 3850 (LJ units), which corresponds to about 2700 in reduced units. This isomorph is generated using the analytical expression for Lennard-Jones potentials as described in Ref. [9] (using the same reference density 1.6 but a slightly lower value of $\gamma$ at the reference density, 4.58 instead of 4.59) and simulated for $10^{8}$ time steps per state point.

\section{GLASS ISOMORPHS}

The temperatures chosen for starting isomorphs are 0.55 and 0.1 . For generating glassy isomorphs a configuration is 
TABLE I. Thermodynamic data for the starting points of glassy isomorphs, obtained by cooling at constant pressure $P=10.0$ from temperature 1.0 over $10^{8}$ steps of size $d t=0.0025$. The cooling rate is therefore $1.8 \times 10^{-6}$ for cooling to $T=0.55$ and $3.6 \times 10^{-6}$ for cooling to $T=0.1$.

\begin{tabular}{|c|c|c|c|c|c|c|c|c|c|}
\hline \multicolumn{5}{|c|}{$T_{\text {start }}=0.55$} & \multicolumn{5}{|c|}{$T_{\text {start }}=0.10$} \\
\hline$\rho$ & $T$ & $P$ & $R$ & $\gamma$ & $\rho$ & $T$ & $P$ & $R$ & $\gamma$ \\
\hline 1.265 & 0.550 & 9.35 & 0.955 & 4.950 & 1.324 & 0.100 & 9.75 & 0.824 & 5.011 \\
\hline 1.278 & 0.577 & 10.68 & 0.954 & 4.971 & 1.337 & 0.105 & 11.21 & 0.834 & 5.002 \\
\hline 1.291 & 0.606 & 11.99 & 0.962 & 5.078 & 1.351 & 0.110 & 12.79 & 0.843 & 4.953 \\
\hline 1.304 & 0.637 & 13.72 & 0.960 & 5.033 & 1.364 & 0.116 & 14.48 & 0.855 & 4.944 \\
\hline 1.317 & 0.669 & 15.37 & 0.965 & 5.015 & 1.378 & 0.121 & 16.29 & 0.864 & 4.916 \\
\hline 1.330 & 0.702 & 16.99 & 0.968 & 4.936 & 1.392 & 0.127 & 18.22 & 0.873 & 4.879 \\
\hline 1.343 & 0.737 & 18.94 & 0.972 & 4.927 & 1.406 & 0.134 & 20.29 & 0.879 & 4.873 \\
\hline 1.356 & 0.773 & 21.07 & 0.973 & 4.874 & 1.420 & 0.140 & 22.49 & 0.886 & 4.829 \\
\hline 1.370 & 0.811 & 23.09 & 0.976 & 4.901 & 1.434 & 0.147 & 24.85 & 0.893 & 4.817 \\
\hline 1.384 & 0.851 & 25.24 & 0.979 & 4.869 & 1.448 & 0.154 & 27.37 & 0.899 & 4.799 \\
\hline
\end{tabular}

drawn from the cooling run close to the desired temperature, and its density is used as the initial state for isomorphs. Due to fluctuations, its density is not necessarily the same as the mean density for the chosen temperature and pressure; similarly, when performing $N V T$ simulations in the glassy state the mean pressure is close to but not equal to the pressure of the cooling run. Table I shows thermodynamic information including the isomorph parameters $R$ and $\gamma$ for the different state point along each of the two isomorphs. We estimate that the starting temperature of our high-temperature isomorph corresponds, if we were to follow the isomorph down to the usual density 1.2 , to a temperature close to $0.42^{1}$. At this temperature the Kob-Andersen mixture can be equilibrated as a liquid, but it requires substantial patience; at the strain rates we apply in our deformation runs, the system can be considered a glassy solid. According to Chattoraj et al. [31], particle displacements become driven more by strain than thermal motion once the strain rate exceeds $10^{-2} / \tau_{\alpha}$. Since our lowest strain rate is of order $10^{-5}$ and $\tau_{\alpha}$ certainly exceeds $10^{3}$, this criterion is met and therefore we can speak of deformation of a glassy amorphous solid, at least regarding steady-state dynamics. Our second isomorph, starting at the lower temperature 0.1 , gives a system deep in the glassy state for which virtually no spontaneous relaxation is expected on conceivable simulation timescales. From Table I we see that the $R$ values for the lower-temperature isomorph are somewhat lower than for the high-temperature isomorph, staying between 0.8 and 0.9 ; one might therefore expect poorer collapse of curves, but we will see that this is not the case for our data.

Starting with the glassy states taken from the cooling run as mentioned above, we ran $N V T$ simulations and then increased the density in steps of $1 \%$ while adjusting the temperature based on the observed value of $\gamma$, according to

$$
T_{n+1}=T_{n}\left[1+\gamma_{n}\left(\rho_{n+1}-\rho_{n}\right) / \rho_{n}\right]
$$

\footnotetext{
${ }^{1}$ This estimation can be done analytically using a formula derived in Eq. (9) of Ref. [9]. The value of $\gamma$ at a reference density must be supplied; we used $\gamma=4.58$ at $\rho_{\text {ref }}=1.6$.
}

This integrates Eq. (4) numerically using the Euler method and when applied to systems in equilibrium generates curves of constant excess entropy. In applying it here we essentially ignore possible complications from being out of equilibrium, assuming, for example, that no significant aging occurs during the simulation. The number of time steps is $10^{7}$ and the starting configuration for each state point is the final configuration from the previous state point. Figure 1 shows three isomorphs in the density-temperature phase diagram including one equilibrium liquid isomorph and the two glassy isomorphs. Figure 2 shows a very good degree of collapse for the radial distribution function along the glass isomorphs when plotted as a function of the reduced pair distance $\tilde{r}=$ $\rho^{1 / 3} r$. This is true even for the high-temperature isomorph, which one might expect to show some (small) changes of structure due to aging [32].

\section{SHEAR DEFORMATION: ANALYSIS OF STRESS-STRAIN CURVES}

When below the glass transition temperature (defined according to the accessible timescales) the system does not undergo any interesting dynamics unless perturbed by some external force. Due to timescale restrictions in simulations, it is easiest to apply a large mechanical deformation to drive the system into a flowing state. In particular, we have chosen to apply simple (Couette) shear at a fixed strain rate and study the stress-strain curve. For shearing we use the SLLOD algorithm combined with Lees-Edwards boundary conditions $[26,33,34]$. When identifying isomorph-invariant properties it is important that the shear rate be specified in an isomorph-invariant way; that is, the reduced-unit strain rate $\tilde{\gamma}=\dot{\gamma}\left(k_{B} T / m\right)^{-1 / 2} \rho^{-1 / 3}$ should be fixed when comparing flowing states at different density-temperature points on an isomorph [12]. The full set of flowing states is therefore characterized by a triple $(\rho, T, \tilde{\gamma})$. Since the physics is in principle invariant along a $(\rho, T)$ isomorph at a given reduced strain rate, we have thus a two-dimensional phase diagram, where a state can be labeled by the isomorph and reduced strain rate. This has been previously shown in the nonviscous regime for the Lennard-Jones fluid [12], but has not been 

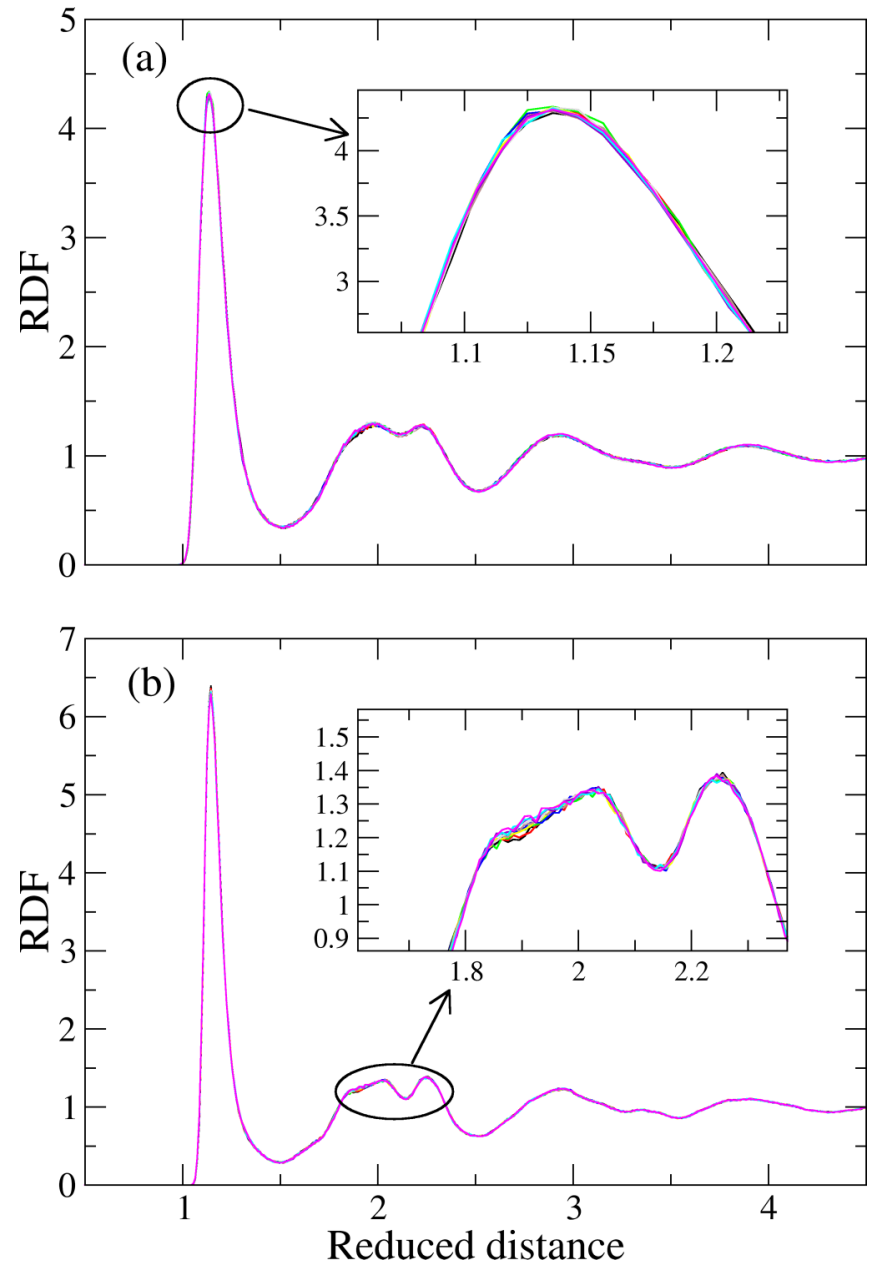

FIG. 2. Radial distribution function (RDF) for the large (A) particles in reduced units along glassy isomorphs starting at (a) $T=0.55$ and (b) $T=0.1$. Each figure shows ten curves, where the density is increased by $1 \%$ for each state point, giving a $9.4 \%$ change in density overall; the temperature increases by $54 \%$ overall. The insets show close-ups of (a) the first peak and (b) the second peak, where very some small deviations are discernible.

tested below the glass transition. In our simulations we choose nominal strain rates of $10^{-2}, 10^{-3}, 10^{-4}$, or $10^{-5}$ and nominal time step of 0.004. By "nominal" time step and strain rate we mean the value in real units at the first point of each isomorph. These values are scaled to keep the reduced-unit time step and strain rate (SR) fixed along the isomorphs. For all our deformation runs we simulated $10^{8}$ molecular dynamics steps, which for the above nominal strain rates give total strains of 4000, 400, 40, and 4, respectively. Chattoraj et al. found that total strains of up to 13.0 or even 24.0 were necessary for accurate statistics [31]. This suggests that our runs are sufficiently long except possibly for the lowest strain rates. Note that the strain itself is dimensionless and therefore does not need to be put into reduced units.

Isomorph theory predicts the whole stress-strain curve to be invariant along isomorphs when stress is given in reduced units $\tilde{\sigma}=\sigma / \rho k_{B} T$. In the small systems typically studied in simulations and particularly at low temperatures and strain rates, however, stress-strain curves in the glassy
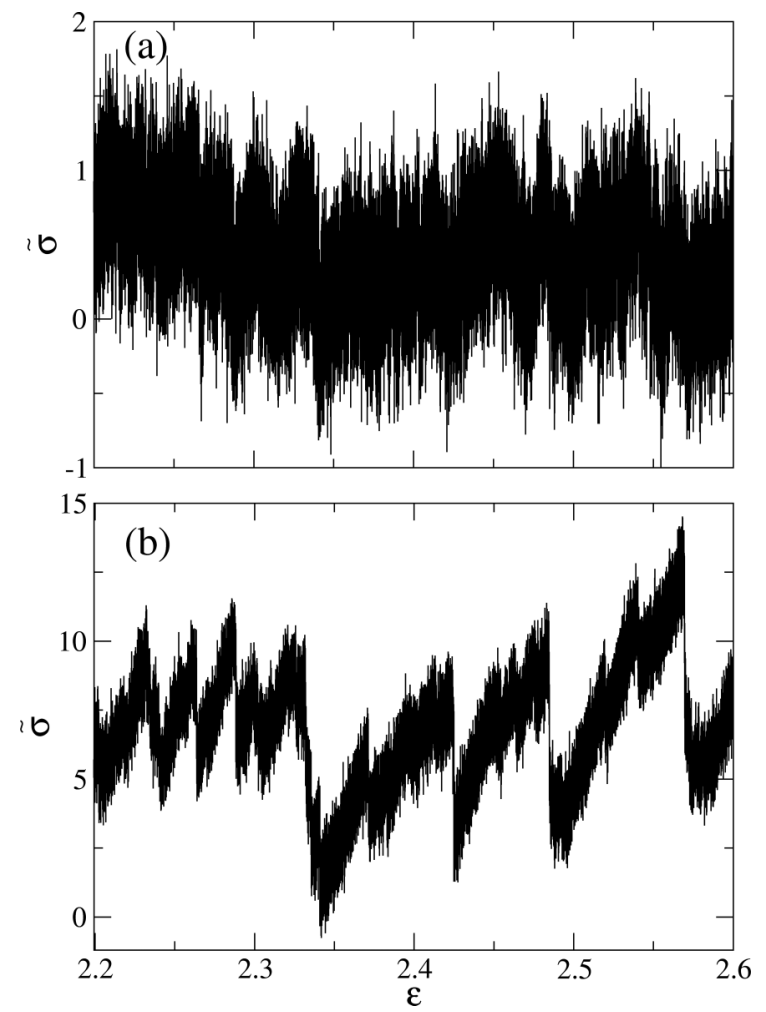

FIG. 3. (a) Section of the stress-strain curve for the lowestdensity state point on the higher-temperature isomorph ( $\rho=$ $1.265, T=0.550$ ) at the lowest nominal strain rate $10^{-5}$. (b) Section of the stress-strain curve for a state point on the lower-temperature isomorph $(\rho=1.324, T=0.100)$ at the lowest strain rate $10^{-5}$. The abrupt drops can be identified with avalanches of plastic activity. The difference in vertical scale between (a) and (b) can be attributed partly to the definition of reduced units for stress.

regime exhibit extremely intermittent behavior [35-38] which is sensitive to initial conditions and other sources of randomness. Examples of this can be found in Fig. 3. Therefore, a collapse of the actual stress-strain curves cannot be expected, except perhaps the initial part which covers the elastic regime and the transition to a flowing state. Instead we choose to study the statistical properties of the steady-state region where properties become time independent, apart from fluctuations. We consider the steady state to have been reached after a strain of 0.5 [39].

The most basic statistical measures that can be extracted from the stress-strain curve are the mean value of the stress (the flow stress) and its standard deviation. Figure 4 shows these quantities plotted in reduced units along the two isomorphs studied, with different nominal strain rates. The curves are consistent with being all flat within the statistical error (for the flow stress at the highest strain rate and the low-temperature isomorph a small systematic decrease with increasing density can be detected, not visible on the scale of the figure). The errors are rather large for the standard deviation at the lowest strain rates where, as we noted above, the total strain is significantly smaller. This figure demonstrates isomorph invariance, the focus of this work. We can also comment briefly on the strain rate and isomorph 

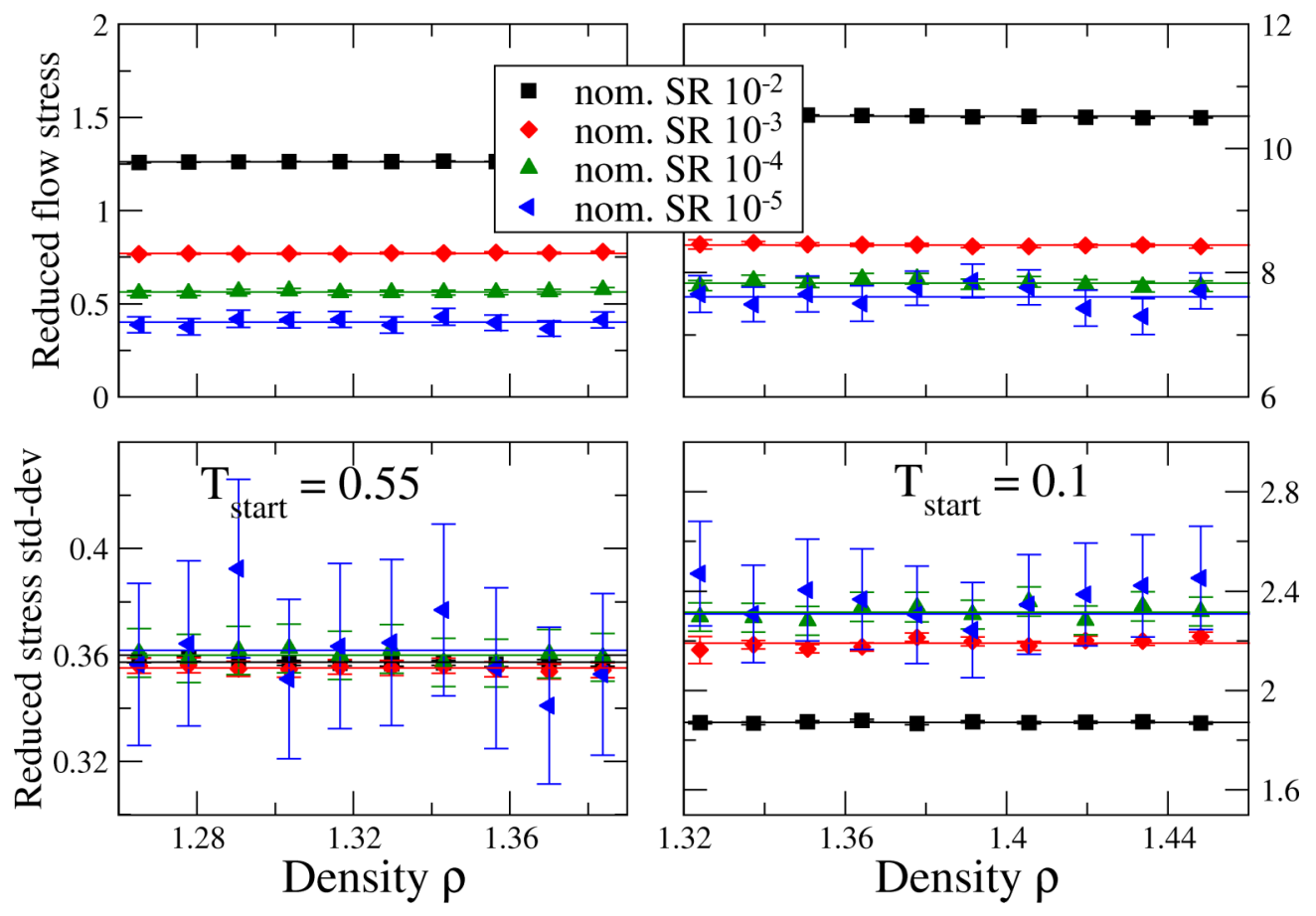

FIG. 4. Flow stress and standard deviation during the steady-state regime as a function of density along high-temperature isomorphs (left panels) and low-temperature isomorphs (right panels) for different strain rates. The legend indicates the nominal strain rates, that is, the real strain rates at the first point on each isomorph; for other state points in each data set, the reduced unit strain rate is the same. Error bars have been calculated as the error of the mean and of the standard deviation, assuming the data are normally distributed [see Eq. (4.14) and Appendix E of Ref. [40]]. We choose the effective number of data points to be the total steady-state strain divided by 0.05 ; we believe data points are statistically independent for strain increments of 0.05 . The horizontal lines indicate the mean value for the isomorph.

dependence. The dependence of flow stress on strain rate is relatively weak given three orders of magnitude variation in the latter. Equivalently, the shear viscosity varies by several orders of magnitude, indicating we are in a strongly nonNewtonian (shear-thinning) regime, as expected for glassy systems [41,42]. Comparing the two isomorphs, the reduced flow stress is a almost factor of 10 smaller at the hightemperature isomorph compared to the low-temperature one, partly reflecting its proximity to the supercooled liquid state, but to some extent also an effect of our choice of reduced units (see Sec. VII below for a discussion of alternative choices). Interestingly, for the high-temperature isomorph the fluctuations of the stress are independent of the strain rate (as well as being invariant along the isomorph). This must mean that the fluctuations here are essentially thermal in origin, despite the rheology being clearly glassy in this regime (as determined from the strain rate dependence of the flow stress).

To investigate the dynamical correlations present in the stress-strain curves and check these for isomorph invariance, we consider the autocorrelation function of the shear stress, plotted as a function of strain interval. Figure 5 shows the results. The collapse is not as good as we have seen in the flow stress. While the curves are somewhat noisy, inspection of the curves shows a trend where the decorrelation moves to lower strain intervals as density increases along the isomorph. To illustrate this more clearly, we fit the autocorrelation curves to a compressed exponential

$$
C(\Delta \varepsilon)=A \exp \left[-\left(\Delta \varepsilon / \varepsilon_{c}\right)^{\beta}\right]
$$

where $\beta$ is greater than unity. For $\beta<1$ this function is known as a stretched exponential, typically used to fit timedependent relaxation correlation curves in the dynamics of supercooled liquid. The characteristic strain $\varepsilon_{c}$ corresponds to the relaxation time $\tau$ in time-dependent correlation functions, indicating roughly the strain interval after which a stress fluctuation has decayed away. As shown in Fig. 5, the compressed exponential can fit the main part of the decay reasonably well, but not the initial slow decay or the negative portion at long times, with values of the characteristic strain $\varepsilon_{c}$ falling in the range $0.01-0.035$ and values of the compression exponent $\beta$ in the range 1.3-1.5. Figure 6 shows that along the isomorphs, $\varepsilon_{c}$ decreases approximately linearly as density increases, in a similar manner for both isomorphs, while $\beta$ increases slightly for the high-temperature isomorph but shows little variation on the low-temperature isomorph. Comparing different strain rates, both $\varepsilon_{c}$ and $\beta$ decrease as strain rate decreases, although for $\beta$ the effect is weak compared to noise. Further investigation with longer runs will be necessary to determine if the apparent variation of $\varepsilon_{c}$ is an artifact of insufficiently long runs, a sign of an imperfect procedure for generating isomorphs, or a genuine limit of isomorph invariance (which is never exact). We note also that there seems to be a systematic undershoot to negative correlation, after most of the stress has decorrelated. This could tentatively be interpreted as a sign of avalanche-type dynamics (discussed below).

As a further type of statistical analysis of the stress-strain curves we attempt to infer something about the microscopic processes by considering the distributions of stress changes 

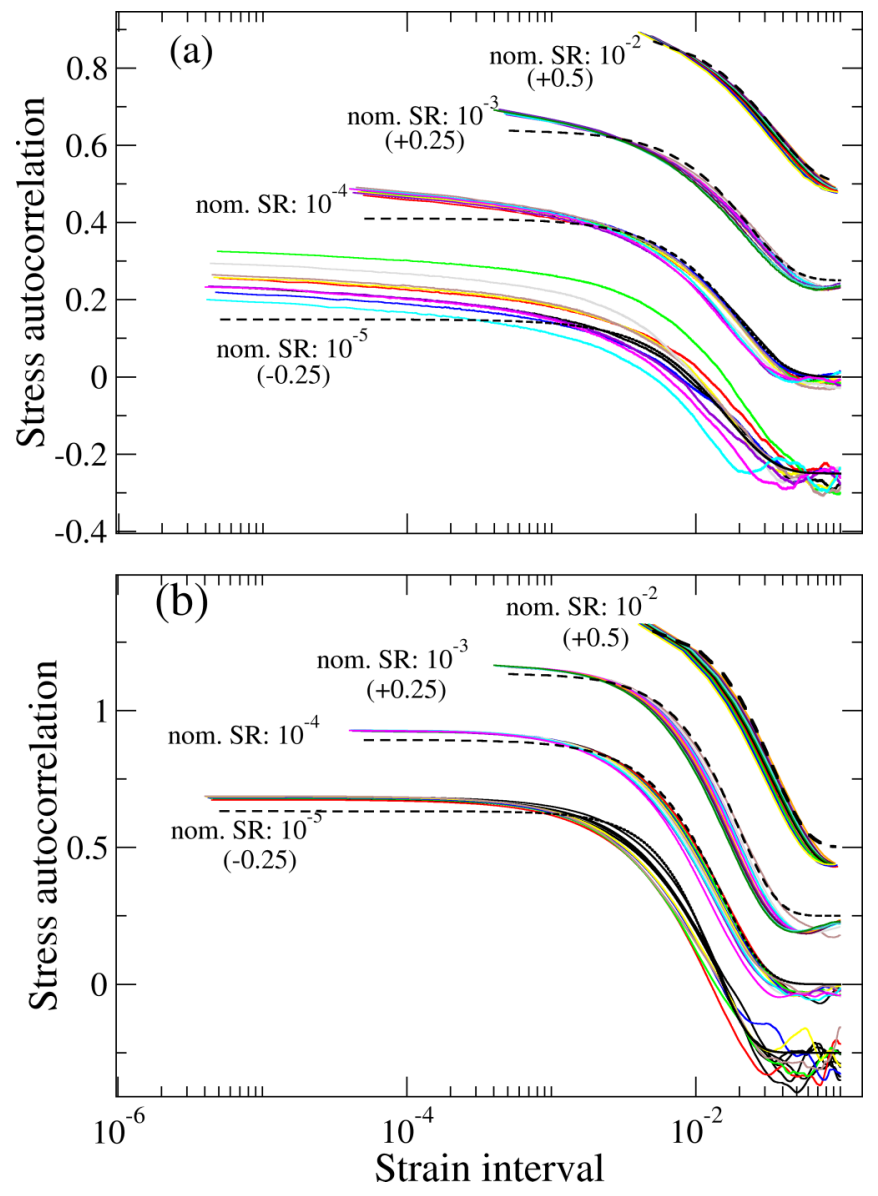

FIG. 5. Normalized shear stress autocorrelation functions along the (a) high- and (b) low-temperature isomorphs for three different strain rates. Curves have been shifted for clarity. The dashed lines indicated fits using a compressed exponential function for the first curve in each set (lowest density and temperature); the parameters can be seen in Fig. 6 .

$\Delta \sigma$ over a given interval of strain $\Delta \varepsilon$. Unlike the case of athermal, infinitely slow driving that has been studied by several authors $[15,35-38,43,44]$, it is not possible to unambiguously identify single flow events or so-called avalanches, since thermal fluctuations tend to merge them together. Lemaître et al. have shown, however, that the dynamics of a glassy system can still be understood in terms of avalanche-type behavior at relatively high temperatures, up to around $0.75 T_{g}[31,45]$. This would put our high-temperature isomorph outside the avalanche-dominated regime and our low-temperature one well within it. Indeed, visual inspection of the stress-stress curves for lower strain rates and temperatures shows drops in the stress reminiscent of avalanche behavior [see Fig. 3(b)]. The distribution of stress changes over a given strain interval can be used to identify signatures of avalanche behavior without having to identify precisely when avalanches occur.

Figures 7 and 8 show histograms of the reduced unit stress changes $\Delta \tilde{\sigma}=\Delta \sigma / \rho k_{B} T$, for different strain intervals $\Delta \varepsilon$ and different strain rates, from simulations on the high- and low-temperature isomorphs, respectively. Curves of the same color represent data from different state points on the isomorph and the near collapse shows that the statistics as probed by these histograms are isomorph invariant to a high degree.
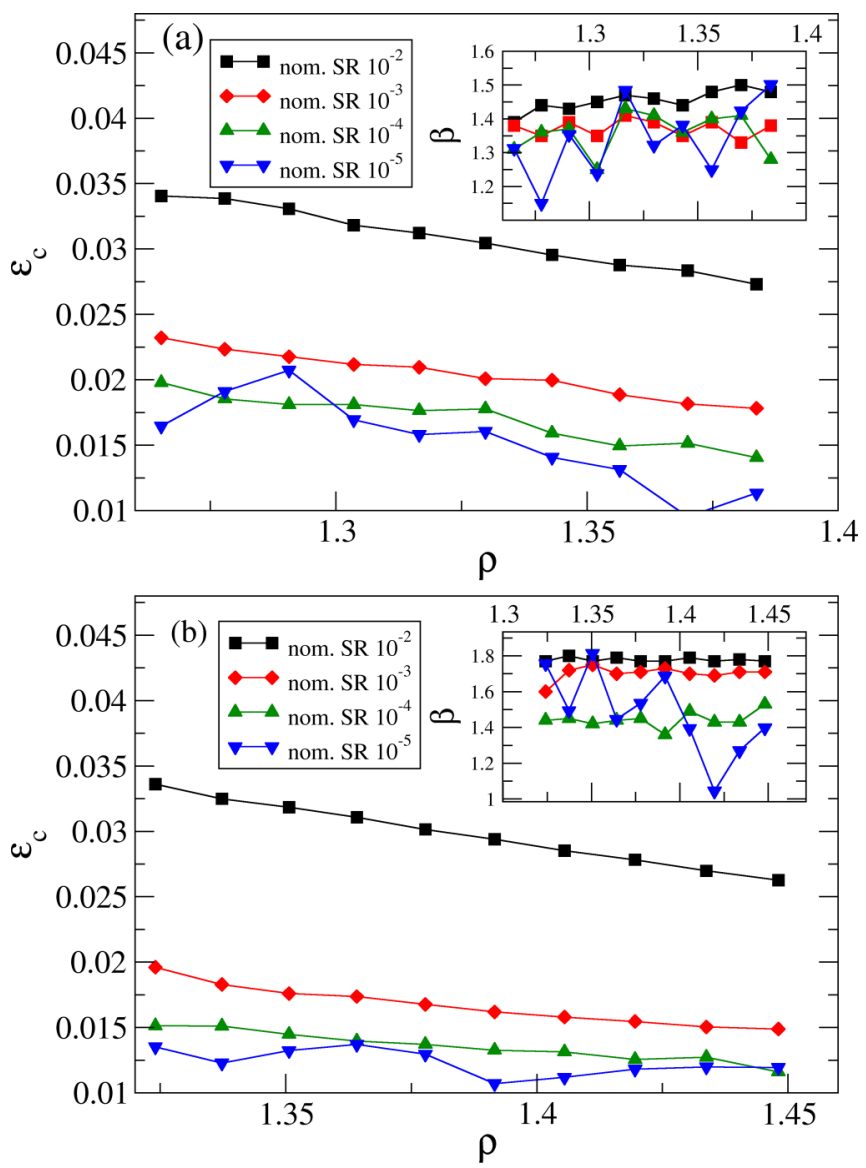

FIG. 6. Fits of shear stress autocorrelation to Eq. (6) shown as functions of density along the (a) high- and (b) low-temperature isomorphs. The characteristic strain over which decays occurs, $\varepsilon_{c}$, decreases approximately linearly as density increases.

This can be seen more explicitly in the insets of Figs. 7(d) and $8(\mathrm{~d})$, where the distributions for the different members of the corresponding isomorph are shown in different colors, for one particular strain interval. Having demonstrated isomorph invariance, it is interesting to note some of the other features of these data. One feature common to both isomorphs and all strain rates is that for sufficiently large $\Delta \varepsilon$, over 0.05 , the histograms converge to a Gaussian whose variance is twice that of the stress fluctuations (mostly within 1\%, 10\% for the slowest two strain rates at the lower temperature isomorph, where the statistical errors are larger). This is expected since our analysis of the autocorrelation indicates that correlations vanish by strain 0.05 in all cases (see Fig. 5) (the characteristic strain interval for decay is between 0.015 and 0.035 , with the functions essentially reaching zero by 0.05 ). For smaller intervals $\Delta \varepsilon$ the distribution is generally narrower and reflects contributions to stress fluctuations from the mechanical driving as well as from thermal fluctuations. As noted above, these cannot be necessarily separated, but a reasonably clear picture emerges from considering the dependence on the isomorph, strain rate, and $\Delta \varepsilon$.

Focusing first on the high-temperature isomorph, Figs. 7(a)-7(d) show stress change histograms for strain rates $10^{-2}, 10^{-3}, 10^{-4}$, and $10^{-5}$, respectively. For all strain rates the distribution converges to the same Gaussian at large 

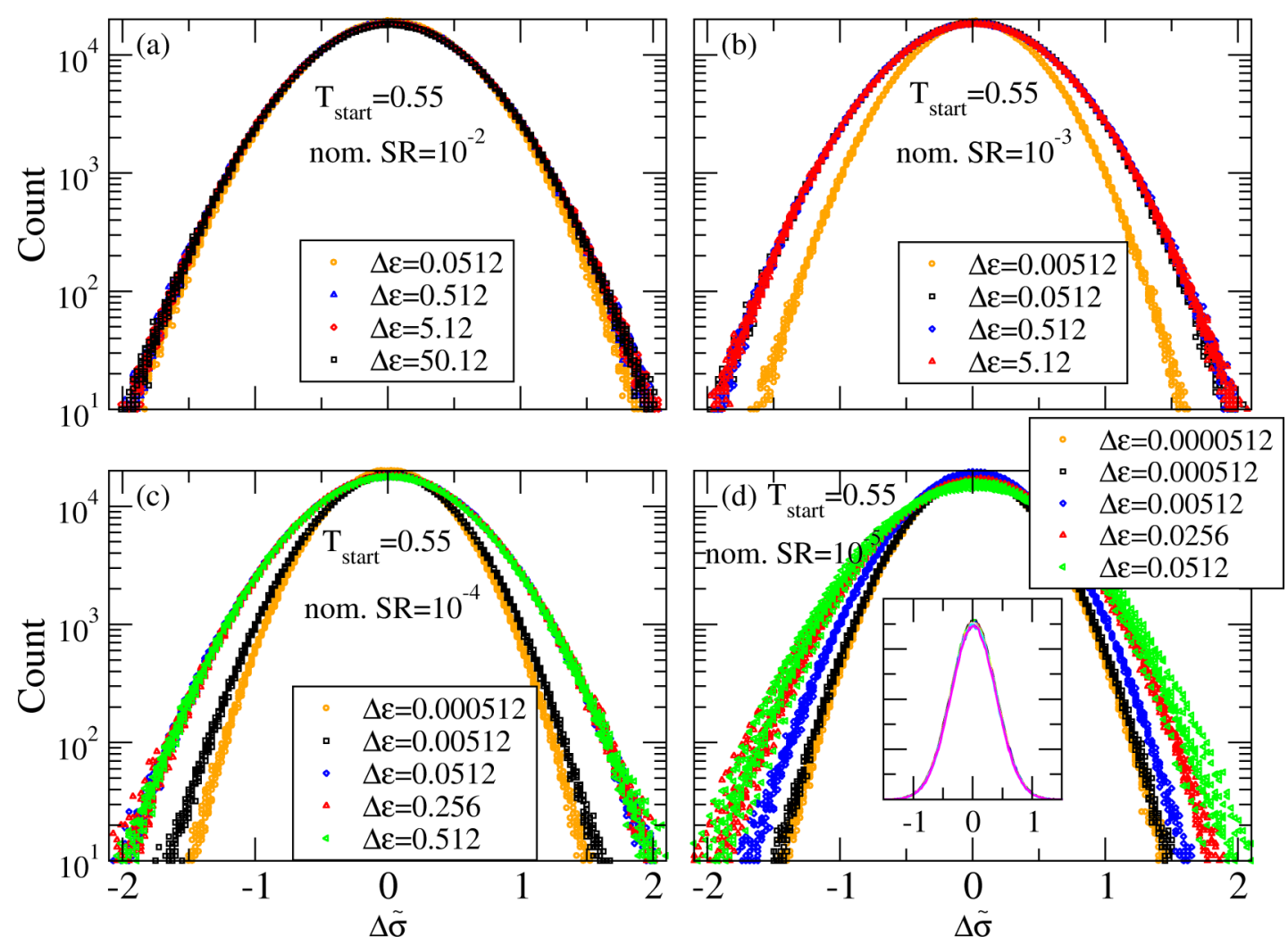

FIG. 7. Histograms of stress changes of intervals as indicated for the high-temperature isomorph for different strain rates. For each strain rate and $\Delta \varepsilon$, distributions from the ten members of the isomorph are plotted in the same color. The fact that they appear as one curve for each color, apart from broadening due to statistical noise at the lowest strain rates, indicates a high degree of collapse. The distributions are essentially Gaussian for all strain rates and strain intervals $\Delta \varepsilon$, and their widths are relative insensitive to $\Delta \varepsilon$ even at the lowest strain rates, indicating that most of the fluctuations are thermal rather than strain driven. The inset in (d) shows an alternative way of exhibiting isomorph invariance for $\Delta \varepsilon=0.000512$, by coloring different members of the isomorph differently, and on a linear scale.

intervals $\Delta \varepsilon$. This is consistent with the bottom right panel of Fig. 4, which showed that the fluctuations of the stress-strain curve are independent of the strain rate (as well as being isomorph invariant) in the high-temperature case, a sign that the fluctuations are dominated by thermal noise in this regime. For the high strain rate the shortest interval is 0.05 , so we see no dependence on the interval here. Some dependence on the strain interval can be seen at low strain rate where the width of the distribution appears to converge to a lower value in the limit of small strain intervals. The timescale for the shortest interval is of order 5 Lennard-Jones units (at the lowest-density point on the isomorph), which should be still somewhat longer than the vibrational timescale; therefore this apparent limit presumably represents the full thermal contribution to the fluctuations for an undeformed glassy system. The increased width at high intervals can therefore be interpreted as coming from the sampling of different glassy configurations due to deformation ${ }^{2}$.

\footnotetext{
${ }^{2}$ Note that this would presumably also happen even without any deformation by waiting long enough for liquid dynamics to become relevant; in that case time, rather than strain, becomes the relevant parameter.
}

Figure 8 shows histograms for the lower-temperature isomorph and the same nominal strain rates as Fig. 7. More interesting behavior is apparent at these low temperatures, particularly at the lowest strain rates, for example (nominal) $10^{-5}$ : For the shortest intervals we see a Gaussian, representing purely thermal fluctuations, which are small at this temperature. In other words, for a strain interval of 0.00005 the stress change due to driving is hidden by the thermal fluctuations. As discussed above, we see a Gaussian at the largest intervals where all correlations have decayed. For intermediate strain intervals, however, a marked deviation from Gaussian behavior appears in the form of a roughly exponential tail on the negative side. This is a clear indication of avalanches: correlated aggregations of multiple microscopic flow events which release the stress, giving large negative stress changes, as studied in the quasistatic case [15,35-38,43,44].

An analysis somewhat similar to ours was carried out by Rottler and Robbins [39], who also found exponential tails at low temperature and strain rate. Note that since we consider a steady-state situation, the mean of the stress changes must be zero, implying that the main Gaussian is shifted slightly to positive values. We have checked this by fitting the Gaussian part (not shown). The positive mean of the nonavalanche fluctuations corresponds to elastic loading which is then released by the avalanches. In the limit of zero temperature and then infinitely slow deformation [37], the narrow Gaussian seen 

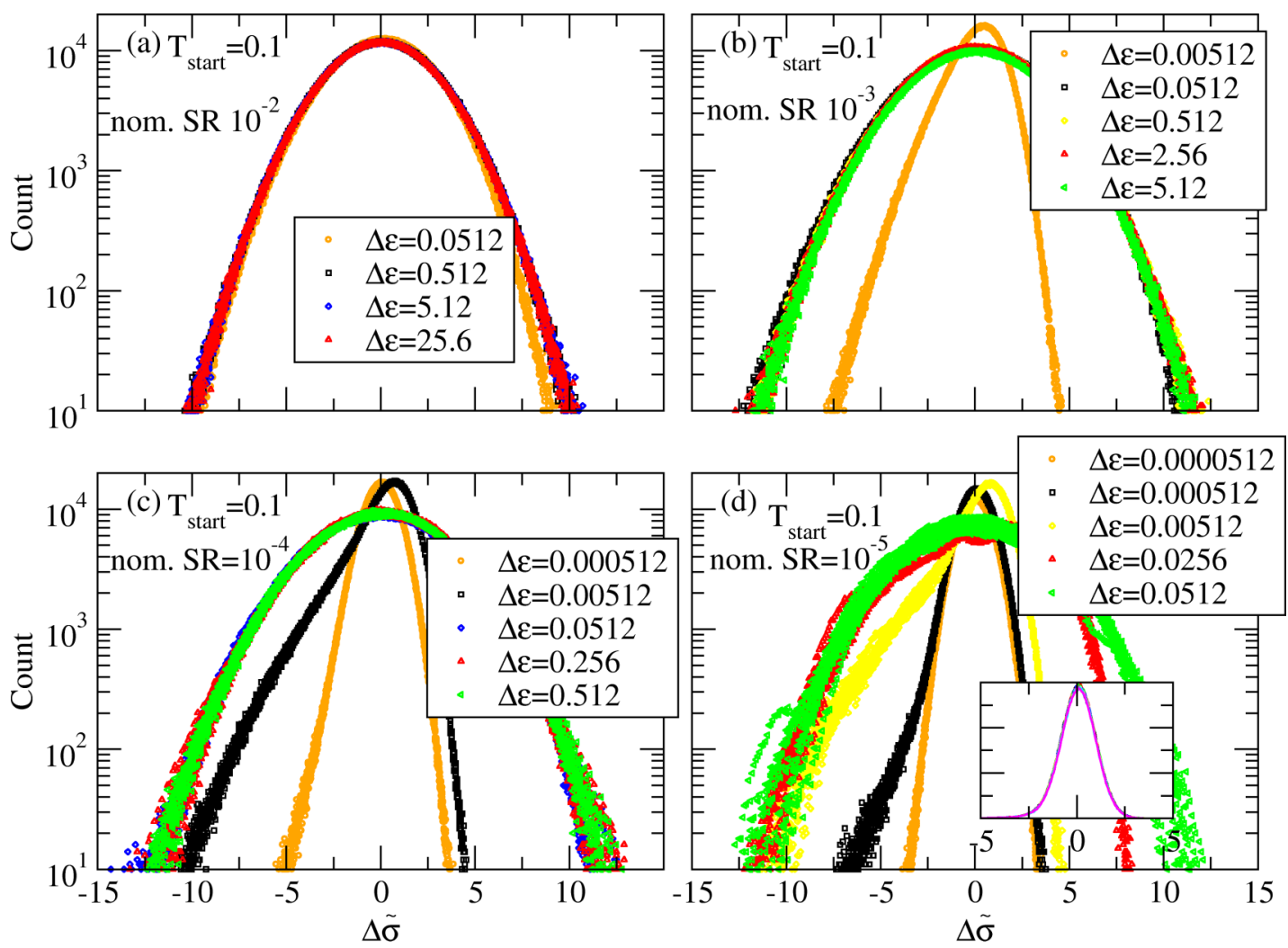

FIG. 8. Histograms of stress changes of intervals as indicated for the low-temperature isomorph for different strain rates. As in Fig. 7, distributions for a given strain rate and strain interval, but different members of the isomorph, are plotted in the same color. They are Gaussian for the largest strain intervals $\Delta \varepsilon$ as well as for the shortest $\Delta \varepsilon$ at the slowest strain rate, where the contribution of strain to the fluctuations is negligible compared to the thermal contribution. For larger $\Delta \varepsilon$ at the slowest strain rate, an exponential tail on the negative side is a clear indication of plastic events organizing into avalanches. For even larger $\Delta \varepsilon$ and at the larger strain rates, mixing of thermal and mechanic noise, and multiple avalanches lead to more disorganized histograms. The inset of (d) shows, on a linear scale, distributions of the second smallest strain interval with the different members of the isomorph represented with different colors as an alternative check of the invariance.

at short intervals would converge to a $\delta$ function at a small positive value (the shear modulus times the strain interval).

The asymmetric deviations from Gaussianity can be quantified by the Fisher-Pearson coefficient of skewness, based on the third moment of the distribution scaled by the cube of the standard deviation

$$
S_{\mathrm{FP}}=\frac{m_{3}}{m_{2}^{3 / 2}},
$$

where

$$
m_{i}=\frac{1}{N} \sum_{n=1}^{N}\left(x_{n}-\bar{x}\right)^{i},
$$

where $\bar{x}$ is the sample mean. Figure 9 shows $S_{\mathrm{FP}}$ as a function of strain interval for four different strain rates for the lowtemperature isomorph. Different curves with the same color come from different members of the isomorph for a given strain rate. The skewness vanishes for short and long strain intervals where, as discussed above, the distributions become Gaussian. The variations between the distributions for a given strain rate are not systematic and thus presumably reflect statistical uncertainty. The variation is relatively small and thus consistent with this measure of the dynamics being isomorph invariant (this follows of course also from the good collapse of the distributions in Figs. 7 and 8). The minimum (most negative) value of the skewness parameter identifies a strain interval $\varepsilon_{s}$ at which the deviation from Gaussianity is most

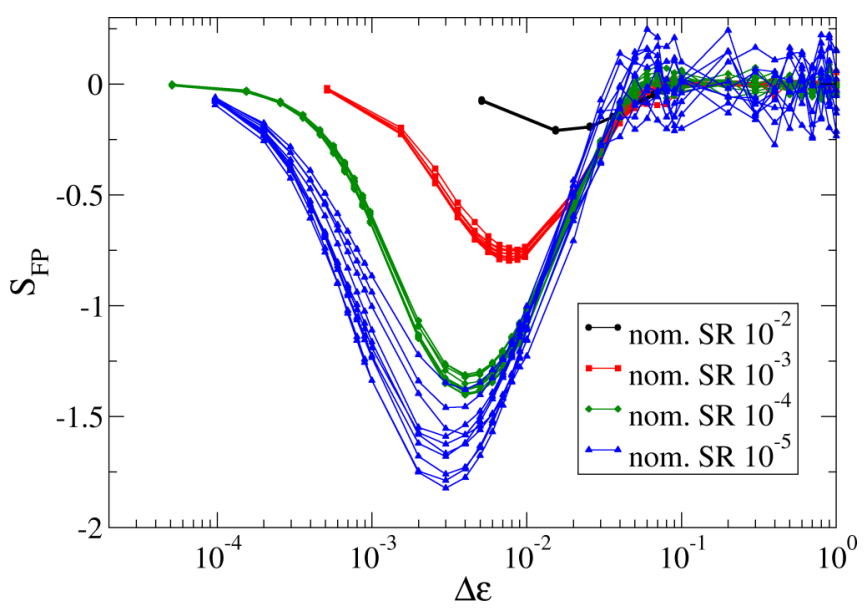

FIG. 9. Fisher-Pearson skewness $S_{\mathrm{FP}}$ of (reduced) stress drop distributions as a function of strain interval for different strain rates for the low-temperature isomorph. Different curves of the same color correspond to different points on the isomorph. 


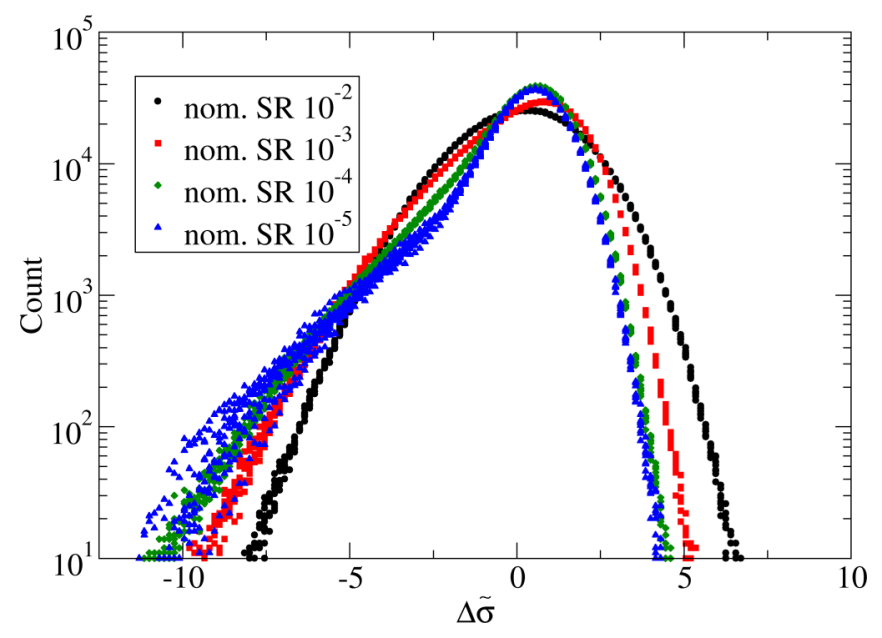

FIG. 10. Histograms of (reduced) stress changes over strain intervals $\varepsilon_{s}$ chosen to minimize skewness for each strain rate, on the low-temperature isomorph. Data for different points on the isomorph are plotted in the same color for each nominal strain rate. In order of decreasing strain, the minimum-skew strain intervals, as judged by eye from Fig. 9, are 0.02, 0.008, 0.004, and 0.003.

pronounced. Histograms for this strain interval are plotted in Fig. 10 for the low-temperature isomorph and different strain rates, with the values of $\varepsilon_{s}$ given. These values are a factor of 2-3 smaller than the characteristic strain intervals identified from the autocorrelation functions (see Fig. 6). For the lowest strain rate it is an order of magnitude larger than the strain interval at which the exponential tail indicating avalanche behavior is seen, $5 \times 10^{-4}$ (see Fig. 8). Denoting the latter by $\varepsilon_{a}$ (where $a$ denotes avalanche), we can tentatively identify, in the low-temperature low strain-rate limit at least, a broad hierarchy of strain scales which characterize different physical processes: (i) the smallest strain scales where stress fluctuations are purely thermal or vibrational; (ii) the avalanche strain $\varepsilon_{a}$ over which stress changes show signs of correlated avalanche-type behavior, of order $5 \times 10^{-4}$; (iii) the strain over which stress change distributions deviate most from Gaussianity $\varepsilon_{s}$ an order of magnitude larger than $\varepsilon_{a}$, where the exponential tails of the avalanches and the changes due elastic loading between them merge to make a broader distribution, but signs of correlation remain; (iv) the characteristic strain $\varepsilon_{c}$ identified via the stress autocorrelation function, where $\varepsilon_{c}$ is of order $2 \times 10^{-2}$, which is a small factor (2-3) larger than $\varepsilon_{s}$; and $(\mathrm{v})$ the strain interval around $5 \times 10^{-2}$ beyond which all correlation has vanished (though this is not physically independent of $\varepsilon_{c}$; rather it represents where the autocorrelation function is small compared to $1 / e$ ).

\section{PARTICLE DYNAMICS UNDER SHEAR: TRANSVERSE DIFFUSIVITY}

As an alternative probe of dynamical processes under steady-state shearing we consider also the particle displacements. Accounting for the contribution to a particle's displacement in the shearing direction when using Lees-Edwards boundary conditions is nontrivial [46], so we consider only the components of a particles displacement transverse to the
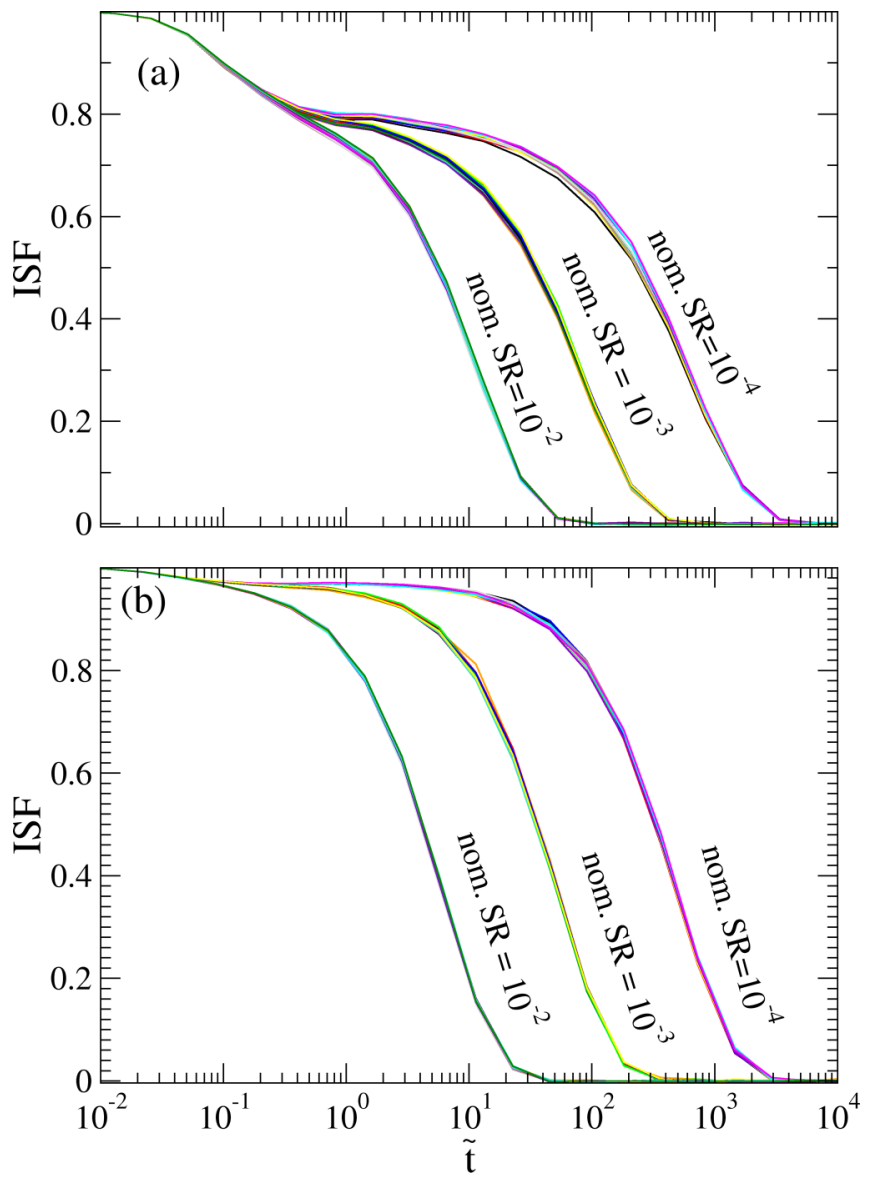

FIG. 11. Self-part of the intermediate scattering function for larger $(A)$ particles based on particle displacements transverse to the shearing direction for (a) the high-temperature isomorph and (b) the low-temperature isomorph, for different strain rates.

shearing direction. Based on these displacements, we compute the self-intermediate scattering function (ISF) and the meansquare displacement (MSD). For the ISF one must choose a wave number $q$, which, as is conventional, we choose to be near the first peak in the structure factor $S(q)$. This must be of course scaled according to $\rho^{1 / 3}$ along an isomorph such that the reduced wave number $\tilde{q} \equiv q \rho^{-1 / 3}$ is constant [this is compatible with choosing $q$ to be near the first peak, as $S(q)$ is invariant in reduced units] [5]. We restrict consideration to the larger $(A)$ particles for brevity. The ISF is shown in Fig. 11.

For both isomorphs and all strain rates we find a good collapse, though slightly less so for the lowest strain rates. Fitting of the curves to a stretched exponential form [Eq. (6), where $\beta<1$ and with $\tau$ instead of $\varepsilon_{c}$ ], not shown, indicates at most a slight systematic variation in relaxation time $\tau$, suggesting the apparent failure to collapse perfectly is mostly due to statistical error. From the fits, for the high-temperature isomorph we find near-exponential behavior $(\beta \simeq 1)$ for the highest strain rates and mildly stretched exponential behavior as the strain rate decreases ( $\beta \simeq 0.85$ at the lowest strain rate). For the low-temperature isomorph we find near-exponential behavior for all strain rates. Stretched exponential behavior is typical of dynamics in the supercooled, highly viscous liquid. The vanishing of stretching, i.e., the near-exponential 

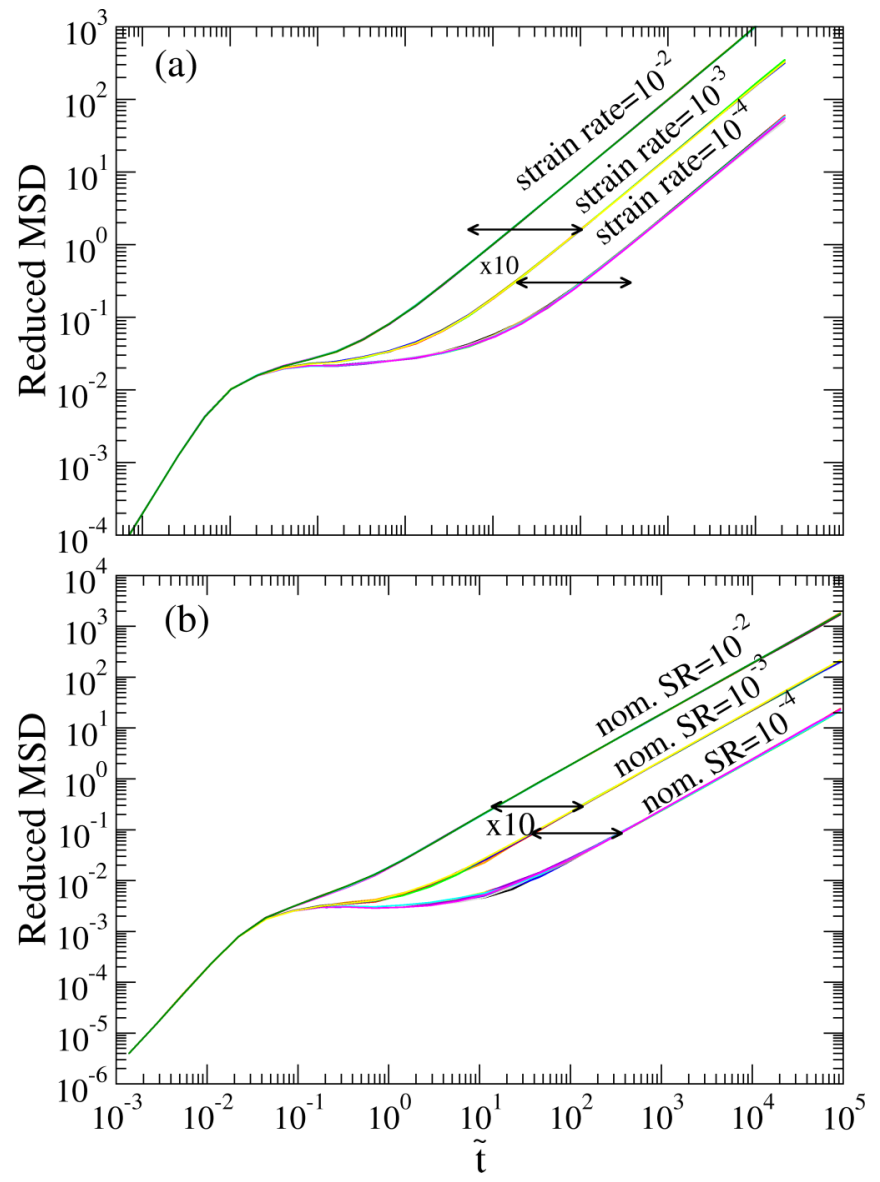

FIG. 12. Mean-square transverse displacement plotted in reduced units for (a) the high-temperature isomorph and (b) the lowtemperature isomorph. The horizontal arrows indicate a factor of 10 in the time axis and can be used to judge by what factor the curves can be shifted onto each other in time.

behavior, at low temperatures and slow shearing indicates that the nature of particle dynamics is different in this regime. Exponential behavior of the self-intermediate scattering function for the same system under shear in the limit of zero temperature was also reported by Berthier and Barrat [41].

Plots of the mean-square transverse displacement in reduced units are shown in Fig. 12. The form of the curves is reminiscent of what is seen for equilibrium viscous liquids: a ballistic regime at short times (where the slope is 2), a plateau of varying extent, followed by a transition to diffusive behavior (slope 1). The collapse is good in all cases, although again some deviations are apparent for the lowest strain rates, particularly around the crossover to diffusive behavior. Superficially, not much difference can be seen between the low- and high-temperature isomorphs, but upon closer examination one can see some physically relevant differences (see Fig. 13). On the higher-temperature isomorph thermal motion is greater; thus the height of the plateau (in units of the interparticle spacing) is larger. More interestingly, in the low-temperature case, the diffusivity curves are essentially a factor of 10 apart in the time axis, corresponding to the factor 10 change in strain rate, while for the high-temperature case the diffusivity curves are shifted by a factor smaller than 10 on the time

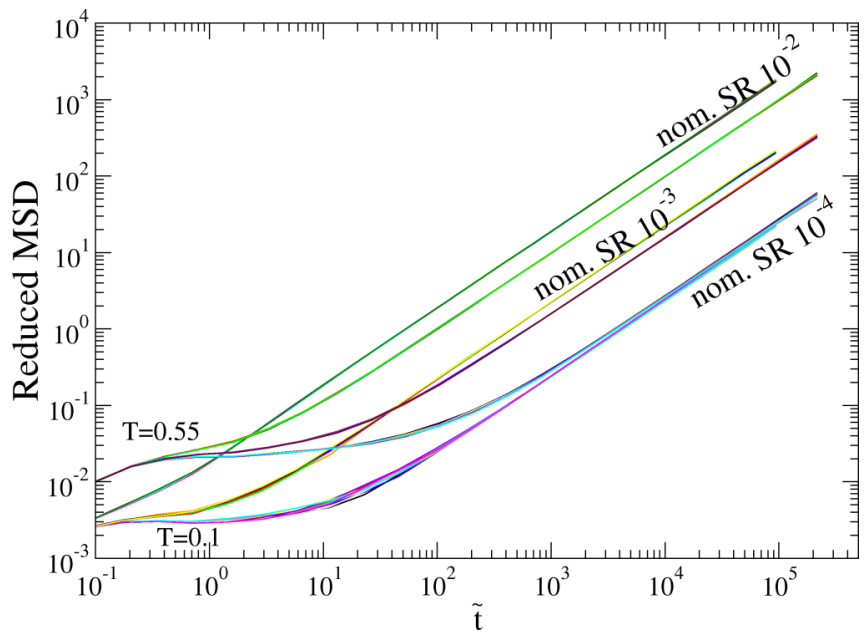

FIG. 13. The MSD curves from Figs. 12(a) and 12(b) plotted together, though without the short-time parts. At low strain rates the MSD appears to become independent of the isomorph, as well as which point on the isomorph. The definition of reduced units means that the curves for the different isomorphs are plotted in terms of different timescales, so caution is required when drawing conclusions from the apparent collapse.

axis. The interpretation is that thermal activation plays a noticeable role in particle diffusion in the high-temperature case, but almost no role in the low-temperature case. In the latter the diffusive motion is determined entirely by the strain rate at the lowest temperatures. Figure 13 emphasizes the long-time parts of the MSD for both isomorphs. In this plot the difference at long times between the two isomorphs appears minimal; the MSD is determined much more by the strain rate than by which isomorph is considered (and almost not at all by which point on the isomorph, which is the essence of isomorph invariance). It must be noted, however, that direct numerical comparison of the MSD curves at different temperatures (isomorphs) for the same nominal strain rate can be difficult to interpret due to the use of reduced units; thus it appears that at nominal strain rate $10^{-2}$ the diffusivity, counterintuitively, is greater on the lower temperature isomorph. Recall, though, that this is in reduced units, i.e., with respect to a timescale defined by the thermal velocity. A meaningful comparison would first of all involve identical reduced (rather than nominal) strain rates; the reduced strain rates for the low-temperature isomorph are a factor of 2.3 higher than the corresponding ones for the high-temperature isomorph. Second, there is a further complication already alluded to, which will be discussed further below, namely, that the definition of reduced units is not unique and a different choice could in principle be more relevant, and elucidate the physics better, in the limit of low temperatures. We emphasize that the most important result in this section is the near perfect collapse of the MSD for different state points along a given isomorph (and given reduced strain rate), when reduced units are used.

Lemaître and co-workers have studied over several papers the effect of finite temperatures and strain rates on avalanche dynamics $[31,45,47]$. They found that studying transverse particle diffusivity is useful for disentangling the effects of strain 
and temperature. In particular, Chattoraj et al. [31] used the transverse diffusivity $D$ determined from the long-time limit of the MSD curves and its strain-normalized analog $D / \dot{\gamma}$. Their Fig. 5 shows nicely the crossover from strain-dominated to temperature-dominated diffusion. As they pointed out, the strain-normalized diffusivity is the more relevant one in the strain-driven regime (low temperatures and strain rates), while normal diffusivity is relevant at high temperatures. Moreover, they showed that the crossover strain rate as a function of temperature tracks more or less the inverse relaxation time: Strain begins to have a pronounced effect on particle diffusion once the strain per relaxation time exceeds an amount of order $10^{-3}-10^{-2}$. Our results are consistent with theirs in terms of the interplay of strain-driven and thermal contributions to particle motion. They did not consider density as a parameter, but our results show that it can be simply accounted for through isomorph invariance and by remembering that "at high temperature" really means "on high-temperature isomorphs."

\section{DISCUSSION}

\section{A. Implications for theories for flow stress}

Several authors have studied the dependence of flow stress of simulated amorphous solids below the glass transition on thermodynamic parameters such as density, temperature, and strain rate and system size $[18,31,39,48,49]$. System size becomes relevant for the flow stress at the lowest temperatures where deformation occurs through avalanches [47]. Some of these works have attempted to determine theoretical expressions or scaling forms to account for size, temperature, and strain rate dependence of the rheology of amorphous solids $[31,48,49]$, while only a few have included density as a variable [18]. One of the crucial implications of the existence of isomorphs is that it does not make sense to think of temperature dependence in isolation from density dependence. We therefore hope that future theoretical work on the rheology of amorphous solids will take this into account. To illustrate this point we consider the expression developed in Ref. [31] for the flow stress as a function of $T$ and $\dot{\gamma}$,

$$
\sigma(\dot{\gamma}, T)=A_{0}+A_{1} \sqrt{\dot{\gamma}}-A_{2} T^{2 / 3}\left[\ln \left(A_{3} T^{5 / 6} / \dot{\gamma}\right)\right]^{2 / 3},
$$

where $A_{0}, A_{1}, A_{2}$, and $A_{3}$ are constants. The form of the expression and the interpretation of the constants were derived through a combination of the theoretical considerations and fitting to data from two-dimensional simulations. We make no claims regarding its validity for three-dimensional situations, but rather wish to illustrate how this expression can be made isomorph invariant in order to ensure consistency with hidden scale invariance. We assume three dimensions in the sense that $\rho$ has units of inverse length cubed. To include density dependence we must allow the $A_{i}$ to be functions of density whose functional form will be determined by isomorph theory. Using standard reduced units (an alternative will be discussed below), we rewrite Eq. (9) in terms of the reduced flow stress

$$
\begin{aligned}
& \tilde{\sigma} \equiv \sigma / \rho k_{B} T \text { and strain rate } \tilde{\gamma}: \\
& \tilde{\sigma}(\dot{\gamma}, \rho, T) \\
& =\frac{A_{0}(\rho)}{\rho k_{B} T}+\frac{A_{1}(\rho)}{\rho k_{B} T} \sqrt{\tilde{\gamma}} \rho^{1 / 6}\left(k_{B} T / m\right)^{1 / 4} \\
& \quad-\frac{A_{2}(\rho)}{\rho k_{B} T} T^{2 / 3}\left\{\ln \left[A_{3}(\rho) T^{5 / 6} \rho^{-1 / 3}\left(k_{B} T / m\right)^{-1 / 2} / \tilde{\gamma}\right]\right\}^{2 / 3}
\end{aligned}
$$

To proceed from here we recall from earlier work [50] that an isomorph in the density-temperature plane may be written as $h(\rho) / k_{B} T=$ const, where the constant indexes the isomorph. The function $h(\rho)$ has not been used so far in the present work; it is the basis of theoretical analysis connecting the shape of the isomorphs to the interatomic potential $[9,50]$. In particular, for any Lennard-Jones system (including mixtures), $h(\rho)$ has the form

$$
h(\rho)=A \rho^{4}-B \rho^{2} .
$$

Sometimes called the density scaling function, $h(\rho)$ describes the way the potential energy surface depends on density. The assumption that this depends only on density (and not on which isomorph one considers) is equivalent to assuming that $\gamma$ depends essentially only on $\rho$. Indeed, $\gamma$ is then simply the logarithmic derivative of $h(\rho)$ :

$$
\gamma(\rho)=\frac{d \ln h(\rho)}{d \ln \rho} .
$$

The normalization of $h(\rho)$ is arbitrary, but it makes physical sense to assume that it has units of energy, since it describes the density scaling of the potential energy surface. If Eq. (10) is to hold in a system with good isomorphs, then the individual terms must be isomorph invariant. Specifically, they must be writable as powers of the combination $h(\rho) / k_{B} T$. Taking the first as an example, we find that $A_{0}=\tilde{A}_{0} \rho h(\rho)$, where $\tilde{A}_{0}$ is a dimensionless constant, i.e., independent of both temperature and density. The full set is, as can be checked straightforwardly,

$$
\begin{gathered}
A_{0}(\rho)=\tilde{A}_{0} \rho h(\rho), \\
A_{1}(\rho)=\tilde{A}_{1} \rho^{5 / 6}[h(\rho)]^{3 / 4}, \\
A_{2}(\rho)=\tilde{A}_{2} \rho[h(\rho)]^{1 / 3}, \\
A_{3}(\rho)=\tilde{A}_{3} \rho^{1 / 3}[h(\rho)]^{-1 / 3} .
\end{gathered}
$$

Inserting these expressions into Eq. (10) yields the following expression for the reduced flow stress as a function of the isomorph scaling combination $h(\rho) / k_{B} T$ and the reduced strain rate:

$$
\begin{aligned}
\tilde{\sigma}(\dot{\gamma}, \rho, T)= & \tilde{A}_{0} \frac{h(\rho)}{k_{B} T}+\tilde{A}_{1}\left(\frac{h(\rho)}{k_{B} T}\right)^{3 / 4} \sqrt{\tilde{\gamma}} \\
& -\tilde{A}_{2}\left(\frac{h(\rho)}{k_{B} T}\right)^{1 / 3}\left\{\ln \left[\tilde{A}_{3}\left(\frac{h(\rho)}{k_{B} T}\right)^{-1 / 3} / \tilde{\gamma}\right]\right\}^{2 / 3} .
\end{aligned}
$$

This is an explicitly isomorph-invariant theoretical expression for the flow stress as a function of $\rho, T$, and $\dot{\gamma}$, based on 
the original expression whose validity was determined (or assumed) for a particular density. However, given that the original expression had a finite limit as $T \rightarrow 0$, it seems problematic that the reduced stress therefore diverges as we consider isomorphs lower and lower in temperature. Therefore, we must consider alternative definitions of reduced units when approaching zero temperature.

\section{B. Alternative reduced units}

Our definition of reduced units, apart from the length unit, is based on thermal motion; thus the energy scale is $e_{0}=k_{B} T$, the velocity scale is $v_{0}=\left(k_{B} T / m\right)^{1 / 2}$, and the timescale is the time for a particle with such a constant velocity $v_{0}$ to cross the interparticle spacing, $t_{0}=\rho^{-1 / 3}\left(k_{B} T / m\right)^{-1 / 2}$. This choice has the advantage of using only macroscopic parameters; apart from the particle mass, no knowledge about the system under consideration (its Hamiltonian, phase diagram, or isomorphs) is needed. However, as noted above, this definition becomes problematic as temperature approaches zero; it is natural at finite temperature but not in the limit of zero temperature, where the thermal timescale diverges. A vibrational timescale which is well defined in that limit is preferable. Noting that the definition of reduced units must satisfy the condition that the reduced quantity is still constant along isomorphs, we can define a new energy scale $e_{1}=e_{0} \frac{h(\rho)}{k_{B} T}=h(\rho)$ and timescale $t_{1}=$ $t_{0}\left(\frac{h(\rho)}{k_{B} T}\right)^{-1 / 2}=\rho^{-1 / 3}[h(\rho) / m]^{-1 / 2}$. These are independent of $T$ and therefore suitable for use in the limit $T \rightarrow 0$. From the interpretation of $h(\rho)$ in terms of the curvature of the pair potential at the nearest-neighbor distance [51], we can interpret $t_{1}$ as a vibrational timescale for a single neighbor pair. Thus we can introduce an alternative reduced stress, denoted by a circumflex,

$$
\hat{\sigma} \equiv \frac{\sigma}{\rho h(\rho)}=\tilde{\sigma} \frac{k_{B} T}{h(\rho)}
$$

and alternative reduced strain rate

$$
\hat{\dot{\gamma}} \equiv \dot{\gamma} \rho^{-1 / 3}[h(\rho) / m]^{-1 / 2}=\tilde{\dot{\gamma}}\left(\frac{h(\rho)}{k_{B} T}\right)^{-1 / 2} .
$$

It is straightforward to rewrite Eq. (17) in terms of the alternative reduced units, giving

$$
\begin{aligned}
\hat{\sigma}(\dot{\gamma}, \rho, T)=\tilde{A}_{0}+\tilde{A}_{1} \sqrt{\hat{\gamma}} \\
-\tilde{A}_{2}\left(\frac{k_{B} T}{h(\rho)}\right)^{2 / 3}\left\{\ln \left[\tilde{A}_{3}\left(\frac{k_{B} T}{h(\rho)}\right)^{5 / 6} \hat{\dot{\gamma}}^{-1}\right]\right\}^{2 / 3} .
\end{aligned}
$$

We thus recover an expression which resembles the original equation (9) while still being explicitly isomorph invariant. We stress that the two expressions [Eqs. (17) and (20)] are equally valid and that for the purpose of checking for isomorph invariance of a quantity the choice of which system of reduced units is not important except for practical purposes, e.g., when $T=0$. However, it can become relevant when comparing different isomorphs in order to identify the relevant physics, or for constructing a theory of the latter, which is evident in the example above. Another example is the comparison of flow stress shown in Fig. 4, where the strong temperature dependence of the reduced flow stress was partly ascribed to our choice of reduced units. Using $\rho h(\rho)$ instead of $\rho k_{B} T$ would probably reduce this variation and is potentially therefore more relevant for the glassy regime. Thus the advantages of one choice over the other are potentially greater clarity, insight, or ease of interpretation.

Lerner and Procaccia studied the flow stress for simulated glasses under steady-state conditions covering both finite temperatures and the athermal limit [18], using a scaling theory based on the approximation of their pair potential by an inverse power law. Their system is modeled using an approximate inverse power law potential, which means that $h(\rho)$ is approximately a power law $\rho^{\gamma}$, in their notation $\rho^{\nu-1}$. Noting that their exponent $v$ corresponds to our $\gamma+1$, all their scaling expressions are in fact compatible with isomorph theory, once one recognizes that their choice of time scaling is equivalent to our alternative reduced units. Another example where the alternative choice of reduced stress was used was the athermal simulations of Ref. [15], where the analysis was based on the isomorph theory and it was assumed (with little discussion) that the correct scaling of the stress at $T=0$ was $\rho h(\rho)$. Our point in the present discussion is that there is a choice of which system of reduced units to use and that that choice is related to how relevant physics is best revealed. It is analogous to the choice of whether we study the standard diffusivity based on mean-square displacement as a function of time or the strain normalized diffusivity based on the meansquare displacement as a function of strain [31]. We note again, however, that using $h(\rho)[9,50]$ is less straightforward than $k_{B} T$ because it depends on the potential and is not directly available in the simulation. In some cases, including the Kob-Andersen system used here, it is known analytically [50,51] [see Eq. (11)]; otherwise it must be identified from the shape of the numerically determined isomorph before conversion into reduced units can take place.

\section{Improvements to future simulations}

Future work in this area could benefit from the following improvements. (i) Our protocol assumes that aging is negligible in our glassy undeformed systems such that it makes sense to generate isomorphs using fluctuations as if in equilibrium. It may be possible to avoid this assumption by using the fluctuations from the steady-state shearing as the next best thing to equilibrium fluctuations. This possibility needs to be developed and evaluated theoretically. (ii) Another route to glassy isomorphs is to use the forces on particles in a single configuration, bypassing the need for equilibrium [52]. (iii) For comparing different isomorphs, consistent reduced strain rates should be used and, moreover, different choices of how to define the reduced units should be considered, as discussed above.

\section{CONCLUSION}

We have simulated isomorphs for the Kob-Andersen binary Lennard-Jones glass and compared their static structure and their dynamics under steady-state shearing deformation. Two isomorphs were generated using the potential energy and virial fluctuations during an $N V T$ simulation (no shear), 
assuming that aging effects could be ignored. This is probably a reasonable assumption for the lower-temperature isomorph, but this is less clear for the high-temperature one, which is only a few percent below the conventional mode-coupling temperature for this system and therefore can be equilibrated as a liquid with longer (but still feasible) simulation times than we have used here. Nevertheless, excellent collapse of the radial distribution function is observed and good collapse for most of the dynamical measures. The worst collapse is observed for the shear stress autocorrelation function, which exhibited a systematic variation of the characteristic decay strain along an isomorph. Better statistics, i.e., longer runs, would probably help, but a more careful determination of the correct isomorph might be necessary, as it could be that this quantity is simply more sensitive to deviations from the correct isomorph than the others we have investigated. Going beyond simply checking for isomorph invariance, we have analyzed the distributions of stress changes over different strain intervals. We showed that different features emerge according to whether purely thermal effects are visible or avalanches, as indicated by an exponential tail in the distribution, or more complex and extremely non-Gaussian distributions at larger strain intervals which include multiple avalanches. Isomorph invariance is clear in all the data presented for this analysis. In comparing the mean-square transverse particle displacements, in addition to almost perfect isomorph invariance, we noted how the MSD curves apparently become independent of temperature in the limit of long times, but also that one has to be careful to compare the same reduced strain rates. We note that no noticeable difference in the quality of the isomorphs is observed, despite the lower-temperature isomorph showing lower values of the correlation coefficient $R$ (see Table I). In the discussion we showed how the existence of isomorphs can inform and constrain the development of analytical theories for how, for example, the flow stress can depend on density, temperature, and strain rate. In addition, an alternative definition of reduced units emerged, the full implications of which are left for future work.

\section{ACKNOWLEDGMENTS}

This work was supported in part by the VILLUM Foundation's Matter Grant (No. 16515). This material is based upon work supported by the National Science Foundation under Grant No. CBET-1804186 (Y.J. and E.R.W.).
[1] N. P. Bailey, T. Christensen, B. Jakobsen, K. Niss, N. B. Olsen, U. R. Pedersen, T. B. Schrøder, and J. C. Dyre, J. Phys.: Condens. Matter 20, 244113 (2008).

[2] N. P. Bailey, U. R. Pedersen, N. Gnan, T. B. Schrøder, and J. C. Dyre, J. Chem. Phys. 129, 184507 (2008).

[3] N. P. Bailey, U. R. Pedersen, N. Gnan, T. B. Schrøder, and J. C. Dyre, J. Chem. Phys. 129, 184508 (2008).

[4] T. B. Schrøder, N. P. Bailey, U. R. Pedersen, N. Gnan, and J. C. Dyre, J. Chem. Phys. 131, 234503 (2009).

[5] N. Gnan, T. B. Schrøder, U. R. Pedersen, N. P. Bailey, and J. C. Dyre, J. Chem. Phys. 131, 234504 (2009).

[6] T. B. Schrøder, N. Gnan, U. R. Pedersen, N. P. Bailey, and J. C. Dyre, J. Chem. Phys. 134, 164505 (2011).

[7] T. S. Ingebrigtsen, T. B. Schrøder, and J. C. Dyre, Phys. Rev. X 2, 011011 (2012).

[8] T. S. Ingebrigtsen, T. B. Schrøder, and J. C. Dyre, J. Phys. Chem. B 116, 1018 (2012).

[9] L. Bøhling, T. S. Ingebrigtsen, A. Grzybowski, M. Paluch, J. C. Dyre, and T. B. Schrøder, New J. Phys. 14, 113035 (2012).

[10] A. A. Veldhorst, J. C. Dyre, and T. B. Schrøder, J. Chem. Phys. 141, 054904 (2014)

[11] F. Hummel, G. Kresse, J. C. Dyre, and U. R. Pedersen, Phys. Rev. B 92, 174116 (2015).

[12] L. Separdar, N. P. Bailey, T. B. Schrøder, S. Davatolhagh, and J. C. Dyre, J. Chem. Phys. 138, 154505 (2013).

[13] N. Gnan, C. Maggi, T. B. Schrøder, and J. C. Dyre, Phys. Rev. Lett. 104, 125902 (2010).

[14] J. C. Dyre, J. Chem. Phys. 148, 154502 (2018).

[15] E. Lerner, N. P. Bailey, and J. C. Dyre, Phys. Rev. E 90, 052304 (2014).

[16] J. C. Dyre, J. Phys. Chem. B 118, 10007 (2014).

[17] J. C. Dyre, J. Phys.: Condens. Matter 28, 323001 (2016).

[18] E. Lerner and I. Procaccia, Phys. Rev. E 80, 026128 (2009).
[19] W. Kob and H. C. Andersen, Phys. Rev. Lett. 73, 1376 (1994).

[20] W. Kob and H. C. Andersen, Phys. Rev. E 51, 4626 (1995).

[21] W. Kob and H. C. Andersen, Phys. Rev. E 52, 4134 (1995).

[22] S. Toxvaerd, U. R. Pedersen, T. B. Schrøder, and J. C. Dyre, J. Chem. Phys. 130, 224501 (2009).

[23] T. S. Ingebrigtsen, J. C. Dyre, T. B. Schrøder, and C. P. Royall, Phys. Rev. X 9, 031016 (2019).

[24] L. Costigliola, D. M. Heyes, T. B. Schrøder, and J. C. Dyre, J. Chem. Phys. 150, 021101 (2019).

[25] T. B. Schrøder and J. C. Dyre, J. Chem. Phys. 141, 204502 (2014).

[26] M. P. Allen and D. J. Tildesley, Computer Simulation of Liquids (Oxford University Press, Oxford, 1987).

[27] U. R. Pedersen, N. P. Bailey, T. B. Schrøder, and J. C. Dyre, Phys. Rev. Lett. 100, 015701 (2008).

[28] S. Toxvaerd and J. C. Dyre, J. Chem. Phys. 134, 081102 (2011).

[29] N. P. Bailey, J. S. Ingebrigtsen, T. S. Hansen, A. A. Veldhorst, L. Bøhling, C. A. Lemarchand, A. E. Olsen, L. Bacher, A. K. Costigliola, H. Pedersen, U. R. Larsen, J. C. Dyre, and T. B. Schrøder, SciPost Phys. 3, 038 (2017).

[30] RUMD software, version 3.3, is freely available at http://rumd. org, Roskilde University, Roskilde, 2018.

[31] J. Chattoraj, C. Caroli, and A. Lemaître, Phys. Rev. E 84, 011501 (2011).

[32] W. Kob and J.-L. Barrat, Eur. Phys. J. B 13, 319 (2000).

[33] D. J. Evans and G. P. Morriss, Phys. Rev. A 30, 1528 (1984).

[34] A. J. C. Ladd, Mol. Phys. 53, 459 (1984).

[35] D. L. Malandro and D. J. Lacks, Phys. Rev. Lett. 81, 5576 (1998).

[36] C. Maloney and A. Lemaître, Phys. Rev. Lett. 93, 016001 (2004).

[37] C. E. Maloney and A. Lemaître, Phys. Rev. E 74, 016118 (2006). 
[38] N. P. Bailey, J. Schiøtz, A. Lemaître, and K. W. Jacobsen, Phys. Rev. Lett. 98, 095501 (2007).

[39] J. Rottler and M. O. Robbins, Phys. Rev. E 68, 011507 (2003).

[40] J. Taylor, An Introduction to Error Analysis: The Study of Uncertainties in Physical Measurements, 2nd ed. (University Science Books, Sausalito, 1996).

[41] L. Berthier and J.-L. Barrat, J. Chem. Phys. 116, 6228 (2002).

[42] T. Voigtmann, Curr. Opin. Colloid Interface Sci. 19, 549 (2014).

[43] E. Lerner and I. Procaccia, Phys. Rev. E 79, 066109 (2009).

[44] E. Lerner and I. Procaccia, arXiv:1004.3193.

[45] J. Chattoraj, C. Caroli, and A. Lemaître, Phys. Rev. Lett. 105, 266001 (2010).
[46] A. Lemaître and C. Caroli, Phys. Rev. E 76, 036104 (2007).

[47] A. Lemaître and C. Caroli, Phys. Rev. Lett. 103, 065501 (2009).

[48] S. Karmakar, E. Lerner, I. Procaccia, and J. Zylberg, Phys. Rev. E 82, 031301 (2010).

[49] H. G. E. Hentschel, S. Karmakar, E. Lerner, and I. Procaccia, Phys. Rev. Lett. 104, 025501 (2010).

[50] T. S. Ingebrigtsen, L. Bøhling, T. B. Schrøder, and J. C. Dyre, J. Chem. Phys. 136, 061102 (2012).

[51] L. Bøhling, N. P. Bailey, T. B. Schrøder, and J. C. Dyre, J. Chem. Phys. 140, 124510 (2014).

[52] T. B. Schrøder (unpublished). 